\title{
QoS hybrid uplink scheduler based on service type for M2M communications in LTE networks
}

\author{
Mariyam Ouaissa ${ }^{1}$, Abdallah Rhattoy ${ }^{2}$ \\ ${ }^{1}$ Information and Communication Systems Engineering Research Group, High School of Technology, \\ Mathematical Modeling and Computer Science Laboratory, Ecole Nationale Supérieure des Arts et Métiers Moulay- \\ Ismail University, Morocco \\ ${ }^{2}$ Department of Computer, Information and Communication Systems Engineering Research Group, \\ High School of Technology, Morocco
}

\begin{tabular}{l}
\hline Article Info \\
\hline Article history: \\
Received Dec 08, 2018 \\
Revised Jan 21, 2019 \\
Accepted Feb 28, 2019 \\
\hline
\end{tabular}

\section{Keywords:}

LTE

LTE-A

M2M

MTC

QoS

Resource allocation

Scheduling

Uplink scheduling

\begin{abstract}
The introduction of Machine-to-Machine (M2M) communications in cellular networks creates a new set of challenges because of the unique service requirements and features of $\mathrm{M} 2 \mathrm{M}$ devices. One of these challenges is the management of radio resources, especially on the uplink because of the unfairness and poor performance that occurs when allocating resources to users. Long Term Evolution (LTE) and LTE-Advanced (LTE-A) are excellent candidates for supporting M2M communications because of their native IP connectivity and scalability for a variety of devices. Therefore, LTE schedulers should be able to meet the needs of M2M devices such as time constraints and specific Quality of Service (QoS) requirements. In this paper, these constraints are studied and analyzed, focusing on three schedulers; they are Round Robin (RR), First Maximum Expansion (FME) and Maximum Throughput (MT). These methods do not provide QoS to users who use different types of traffic flows. The solution proposed in this work is a hybrid model between two schedulers each of them is the best scheduling solution for the real-time service and the other for the non-real-time service, in order to meet QoS criteria maximizing throughput and minimizing packet loss. Video and voip were selected as real-time traffic and best effort for non-real time. The simulations results show that the proposed scheduler reaches the lowest Packet Loss Rate (PLR), delivering highest throughput and goodput among the other strategies.
\end{abstract}

Copyright @ 2019 Institute of Advanced Engineering and Science. All rights reserved.

\section{Corresponding Author:}

Mariyam Ouaissa, Information and Communication Systems Engineering Research Group, High School of Technology, Mathematical Modeling and Computer Science Laboratory,

Ecole Nationale Supérieure des Arts et Métiers Moulay-Ismail University,

Km 5, Rue d'Agouray, N6, Meknès 50000, Morocco.

Email: mariyam.ouaissa@edu.umi.ac.ma

\section{INTRODUCTION}

The Internet of Things (IoT) [1]-[2] refers to the continuous growth of networks of everyday objects that interconnect with each other or with other Internet systems via wireless sensors attached to them. IoT promises a future where billions of smart devices will be connected and managed through a range of communication networks and cloud-based servers, enabling the emergence of a broad spectrum of monitoring and control applications. Machine to Machine (M2M) communications [3] represent the IoT bridge supported by cellular networks. They are also known as Machine Type Communications (MTC) [4] and are a key technology for partially enabling IoT. M2M communication is a new paradigm that facilitates the ubiquitous connectivity between a myriad of devices without or with limited human intervention. 
The growing demand for connectivity has challenged network operators to design new radio resource allocation algorithms to handle the massive scale of MTC at affordable costs [5].

Scheduling or resource allocation is one of the major challenges facing M2M communications over Long Term Evolution (LTE) networks. M2M traffic has unique characteristics; it usually consists of a large number of small data packets, with specific delays, generated by a potentially large number of devices competing on scarce radio resources [6]. Currently, LTE is an internationally recognized mobile communication standard for 4G [7]. With the advent of M2M devices and network capabilities, an appropriate uplink scheduling strategy is strongly needed for such systems. Scheduling method is one of the hot topics among current research for M2M on LTE. There are major differences between M2M communications and Human to Human $(\mathrm{H} 2 \mathrm{H})$ communications [8], [9].

In this paper, an in-depth research on the allocation of M2M communication resources and LTE scheduling is performed and a method for M2M uplink scheduling based on the existing LTE scheduling algorithms is introduced. This proposed scheduler provides increased system throughput and reduces the average system service time by using the appropriate scheduler depending on the type of service Real Time (RT) or Non Real Time (NRT). The rest of the paper is organized as follows: in section 2 we discuss the work already done in this context. Section 3 describe the M2M system architecture, the M2M traffic characteristics and the scheduling process for M2M communications. The fourth section deals with the procedure and the mathematical modeling of the problem of allocation of radio resources in LTE uplink, as well as the algorithms considered in this article. We present our solution proposed in section 5 . We evaluate and analyze in section 6 the performances of the existing algorithms as well as our proposition and we indicate the best scheduler in term of throughput, goodput, fairness index, spectral efficiency and Packet Loss Rate (PLR).

\section{RELATED WORKS}

In this section, we review existing works addressing uplink scheduling issues due to incorporation of M2M in LTE systems. In [10], authors introduce a novel M2M scheduling metric that we term the "statistical priority". Statistical priority is a term that indicates the uniqueness of the information carried by certain data packets sent by Machine Type Communications Devices (MTCDs). If an MTCD data unit is signicantly dissimilar to the previously sent data, it is considered to carry non-redundant information. Consequently, it would be assigned higher statistical priority, and this MTCD should then be given higher priority in the scheduling process.

The authors in [11] propose a Smart Uplink Scheduling Algorithm based on existing LTE cellular network according to the characteristics of M2M services and LTE uplink scheduling. The algorithm integrates the improved Proportional Fairness scheduling algorithm with H2H and M2M service mix queuing model. This algorithm offers an increased system throughput the process and reduces the average delay of system service without affecting into the traditional voice and video communications.

M2M Aware Scheduling Algorithm (M2MA-SA) proposed in [12], the idea behind this solution is to create an algorithm which differentiates between M2M and H2H services. For massive M2M services, a compound 2-phase scheduling mechanism is presented, which combines maximum-utility scheduling with round robin scheduling. For H2H services, an algorithm named Iternative Maximum Expansion (IME) is used which satisfied the contiguity constraint of LTE uplink resource allocation. The simulation results show that M2MASA not only guarantees the QoS of $\mathrm{H} 2 \mathrm{H}$ communication, but also improves the system throughput and the capacity of $\mathrm{H} 2 \mathrm{H}$ users.

The authors of the article [13] introduce, analyze and simulate a new predictive/proactive resource allocation scheme for the LTE uplink for use with event based M2M applications. In this scheme, when one device in a group sends a scheduling request, the eNodeB identifies neighbor devices in the same group which may benefit from a predictive resource allocation in lieu of waiting for those neighbors to send a scheduling request at their next scheduled opportunity.

This paper [14] proves this energy minimized scheduling problem is NP-hard, and proposes a heuristic energy-efficient algorithm to address it. This algorithm effectively schedules the transmissions of an M2M node in the same time slots, so that the active time of the M2M node can be minimized. The experiment results show that under limited bandwidth resource, the algorithm can maintain fairness and low data dropping ratios while achieving energy efficiency for a reasonable number of M2M nodes.

In this article [15], the authors propose an M2M uplink scheduling algorithm that offers a balance between throughput and delay requirements. It is also adaptive to traffic characteristics since it considers both channel state and system deadlines in an adjustable manner according to network needs.

Mukesh Kumar Giluka et al proposed a class based dynamic priority scheduling algorithm for uplink transmission of M2M and $\mathrm{H} 2 \mathrm{H}$ traffic in LTE. The performance of the algorithm is evaluated by

QoS hybrid uplink scheduler based on service type for M2M communications in LTE... (Mariyam Ouaissa) 
various metrics such as $\mathrm{H} 2 \mathrm{H}$ throughput and system throughput and also compared with existing schedulers [16].

This work [17] discusses the challenges of scheduling UEs and M2M terminals in LTE networks. The LTE scheduler plays an important role in distributing radio resources to user equipments (UEs) and M2M terminals. We present a hybrid uplink scheduler that balances the radio resources allocation to preserve human users QoE and fairly fulfills M2M terminals communication requests. The simulation results show that the proposed solution provides fair scheduling of M2M terminals without impacting the UE.

In [18], the authors propose two scheduling schemes for the uplink of LTE based cellular systems, which take into account both the channel conditions and the maximum allowed delay of each device requesting to be served. In contrast to other scheduling algorithms no classes of devices are formed, but the exact delay constraint of each device is considered, approaching the requirements of $\mathrm{M} 2 \mathrm{M}$ communications in a more realistic way. This way, the number of effectively served requests increases, while it becomes possible to exploit the exact delay constraints in order to put the devices in sleep modes, until next transmissions. It is also shown that dividing the devices into a limited number of QoS classes deteriorates the system's performance.

\section{BACKGROUND}

In this section, we describe the network architecture of M2M system, we present the characteristics of $\mathrm{M} 2 \mathrm{M}$ communications and we introduce the M2M scheduling process.

\subsection{System Architecture}

The architecture of a M2M system [19]-[20] includes several parts such as Machine Type Communication Devices (MTCDs), Machine Type Communication Gateways (MTCGs), the communication network and Machine Type Communication Servers (MTC Servers). The MTCD is the device used to collect information from the environment. The MTCD sends data to the base station, which is known as evolved Node B (eNodeB) in LTE, either directly or via an MTCG. The MTCG acts as a cluster head for a group of MTCDs. The MTCG applies some forms of processing on data coming from MTCDs, to compress the amount of data to be sent to the eNodeB. The MTC server is the end-target of the data sent by MTCDs. It receives data via the backhaul from the eNodeB and makes it available for access by human or machine type users through some application in Figure 1 [21], [22].

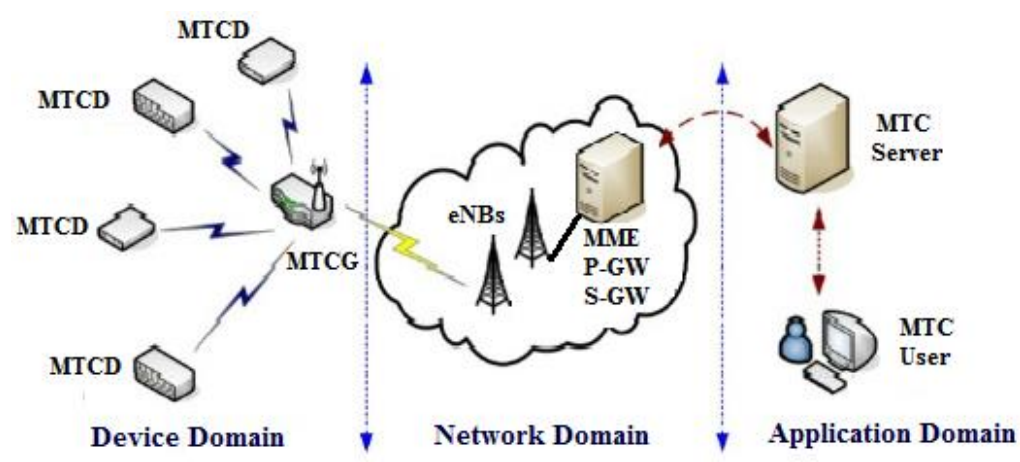

Figure 1. M2M system architecture

One of the important tasks of eNodeB in the LTE network is Radio Resource Management (RRM), which is the acceptance or rejection of network connection requests, ensuring optimal distribution of radio resources between terminals. It consists mainly of two elements Admission Control (AC) and Packet Scheduling (PS). The AC is responsible for the acceptance and rejection of new requests, but the PS realizes the allocation of resources effectively to the various users already accepted by the CA. The AC processes the new requests for connection to the network, the decision to accept or reject a request depends on the network's ability to offer the Quality of Service (QoS) required by this request while ensuring the QoS of the requests already admitted in the system. The PS for its part performs the mapping, that is to say select the users who will use the channel by assigning them radio resources RBs that allow them to maximize the performance of the system [23]. 
There are several parameters to evaluate the performance of the system, for example we can mention: the spectral efficiency (total system throughput), the equity between the users, the waiting time of each user before it is served. The diversity of the performance parameters allowed the creation of several types of schedulers. For the uplink (considered in this article), the method used is Single Carrier Frequency Domain Multiple Access (SC-FDMA), a variant of Orthogonal Frequency Division Multiple Access (OFDMA), they have almost the same performance, but SC-FDMA transmits the sub-bands sequentially to minimize the Peak-to-Average Power Ratio (PAPR), this is necessary because for the uplink direction, the terminal equipment is provided with a battery with limited life. Two other parameters affect the design of LTE Uplink scheduling algorithms. These two parameters are imposed by the access method SC-FDMA, are: the minimization of the power of transmission in order to maximize the life of the batteries of the UEs and the RBs allocated to a single user must be contiguous. This makes the allocation of radio resources for LTE Uplink more difficult than for the Downlink [24]-[25].

\subsection{The Characteristics of M2M Communications}

$\mathrm{M} 2 \mathrm{M}$ communications characteristics differ from those of $\mathrm{H} 2 \mathrm{H}$ communications in several aspects. 3GPP TS22.368 makes a detailed prediction and classification for the prospective M2M services [26]. By analyzing we summarize the main characteristics of the M2M services in LTE as follows:

a. The number of M2M terminals is so huge that it has at least two more orders of magnitude than that of the traditional $\mathrm{H} 2 \mathrm{H}$ terminals.

b. Most of the M2M communications traffic occurs in the uplink direction from the MTCDs to the eNB.

c. M2M terminals initiate sessions frequently, but each session only lasts for a short time.

d. Each M2M session sends only one or a few packets, and the length of each packet is very short.

e. Many MTC applications have strict data transmission deadlines. Abiding by deadlines is necessary to report an alarm for a disaster, to maintain a certain data rate or a certain QoS and to send data before they become useless or obsolete.

f. There are numerous types of MTCDs and they are used in a wide variety of applications. Hence, MTCDs vary widely in terms of requirements of deadlines and needed QoS.

\subsection{The M2M Scheduling Process}

Scheduling is the process performed by the eNodeB to allocate time-frequency resources known as Physical Resource Blocks (PRBs) to human User Equipment (UEs) or MTCDs in downlink [27]-[28] or uplink direction. For uplink scheduling, the assigned resources to a UE or an MTCD must be contiguous [29]. The general scheme of M2M uplink scheduling algorithms can be divided into 2 main steps:

Step 1 (Time Domain Packet Scheduling): In this step, the eNodeB selects an MTCD or a group of MTCDs with the highest priority based on channel state, fairness, delay, QoS, etc, to be scheduled for the current Transmission Time Interval (TTI).

Step 2 (Frequency Domain Packet Scheduling): In this step, the eNB selects the PRBs to be assigned to the terminal that have been selected in the TDPS stage. The selected MTCD is assigned contiguous PRBs based on channel state, taking data rate maximization and fairness into consideration [30].

\section{OVERVIEW OF LTE UPLINK SCHEDULING}

This section provides an overview of uplink scheduling process with mathematical modeling and studies the main scheduling algorithms using in LTE uplink network.

\subsection{Uplink Scheduling Process}

Scheduling is referring to the process of allocating resources to User Equipment based on scheduling algorithms that is located at the LTE base station.

Scheduling is a procedure that allocates physical resources for users according to a certain algorithm in each TTI. Resource Block (RB) is the minimum unit of resource allocation and TTI is 1ms, also RB is a resource grid that consists of 12 subcarriers in one time slot. The frequency resources corresponding to the $\mathrm{RB}$ can be occupied for a period of TTI once one RB is allocated to a user.

When having data to send, a user firstly sends an uplink scheduling request to base station and reports its own cache, which can make the scheduler of base station acquire necessary data and make an accurate decision about scheduling. Then the scheduler sends scheduling results to user. When receiving the permissible message of scheduling, user sends data as required in the scheduling results which specify the transmission format, the available resources and the transmission time. Besides, LTE uplink scheduling should satisfy the constraint that all RBs assigned to a service request are contiguous in frequency domain [31], [32]. 


\subsection{Mathematical Modeling}

The uplink scheduling algorithms take a matrix with $\mathrm{K}$ rows (number of active users) and $\mathrm{M}$ columns (number of RBs). Mi,m is the associated value in UE $\mathrm{i}$ and RB $\mathrm{m}$. Depending on the paradigm used, Channel Dependent (CD) or Proportional Fairness (PF), this value represents the Channel State Information (CSI) of each RB for each UE or the CSI rate report. The values of the matrix represent the association between UE-RB, these values are used by the scheduler in Figure 2.

There are two major CD and PF paradigms in the literature that make it possible to fill the matrix. The first CD, in the process of creating the matrix, Channel State Information (CSI) or the channel state is considered, so UEs that have the highest CSI values will have the chance to allocate more resources, this approach reaches the best values in flow, but she suffers from starvation problem. While the PF, meanwhile, he takes the CSI rate report for each UE. So equity is proportional to the CSI value and we create the matrix. This approach achieves good rates by solving the famine problem at the same time [33].

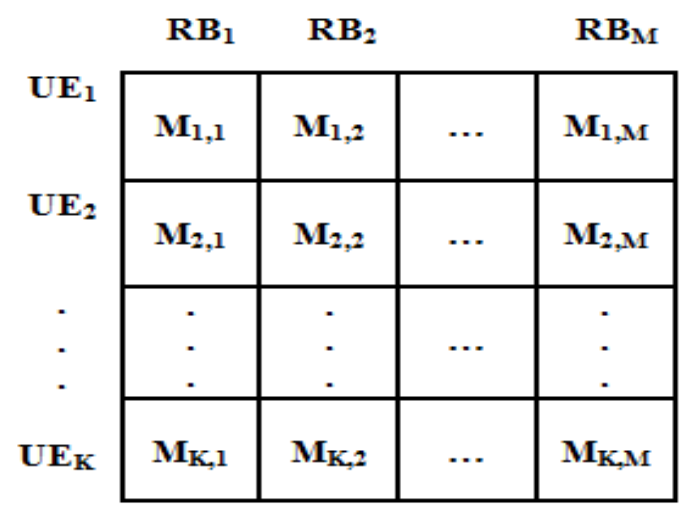

Figure 2. UE-RB association matrix

\subsection{Scheduling Algorithms}

Several approaches and algorithms have been proposed in the literature to answer the need to allocate resources efficiently, this diversity and multitude of algorithms is related to the factors considered allowing the optimal management of radio resource, specifically the type of traffic and QoS requested by the UE [34]-[35].

\subsubsection{Round Robin (RR)}

This is one of the classic schedulers used in many older systems. Its principle is too simple, just distribute the RBs on all the UEs that require radio resources. In our case, it is used as a reference to evaluate other schedulers. The principle of $R R$ is to divide the set of $R B s$ on the UEs, $E\left(\frac{\left|N_{R B}\right|}{\left|N_{U E}\right|}\right)$ to form Resource Chunks (RC), the RC is a subset of RBs, after we distribute RCs across all UEs.

We note that $E\left(\frac{\left|N_{R B}\right|}{\left|N_{U E}\right|}\right)$ is the integer part of the division.

\subsubsection{First Maximum Expansion (FME)}

The idea of this algorithm is to start allocating resources from the largest value in the matrix, then expand the process of allocating resources right and left.The steps of the FME algorithm are:

Step 1: find the pair (UE0,RB0) with the largest value in the matrix.

Step 2: allocated RB0 to UE0.

Step 3: for this user, UE0 compare the values of the matrix for RB+ 1 and that of RB-1.

Step 4: If the value on the right is greater than the one on the left, then widen the allocation to $\mathrm{RB}+1$, otherwise go to RB-1.

Step 5: for each column find the maximum value, allocate this RB to the selected user if it corresponds to the max value of the column or if it is not the max, but the allocation cannot be performed without that the constraint of contiguity be respected.

Step 6: Repeat step 5 until all RBs are exhausted. 


\subsubsection{Maximum Throughput (MT)}

This algorithm provides a maximum overall network throughput by allocating each RB to UEs that experience better quality of the radio channel. That means, a UE that has a very good quality of the channel will always be programmed by the scheduler for the transmission (uplink and/or downlink), the scheduler chooses the user $\mathrm{i}$ for the transmission of the kth resource block by the following formula:

$$
\mathrm{p}_{\mathrm{i}}^{\mathrm{k}}=\arg \max _{\mathrm{i}}\left(\mathrm{d}_{\mathrm{k}}^{\mathrm{i}}(\mathrm{t})\right) ; 1 \leq \mathrm{i} \leq \mathrm{N}
$$

Where: $d_{k}^{i}(t)$ is the expected debit for the user $i$ at time $t$ in the kth resource block and $N$ the number of users in the cell. The disadvantage of this scheduler is that it is not fair when allocating resources for users at the edges of the cell.

\section{OUR PROPOSED SCHEDULER}

In order to find a compromise between throughput and fairness, we propose a new scheduling algorithm that takes advantage of the benefits of the RR scheduler and the FME scheduler to give high priority to real-time traffic. The proposed new scheduler is intended to combine RR and FME schedulers. Depending on the type of traffic flow, it can be said that when the real-time traffic is started, the RR is applied because the real-time traffic flow requires a very high data rate. Otherwise, FME is applied to show equity among other users. The flowchart in the Figure 3 shows the algorithm of the proposed scheduling. Differentiation between real-time and non-real-time occurs at the beginning of the scheduler before checking channel conditions to treat real-time traffic as a special case. This allows us to give higher priority to realtime traffic, so that the result appears in terms of maximum throughput and minimum packet loss. After that, we can apply RR scheduler to real time to get high throughput.

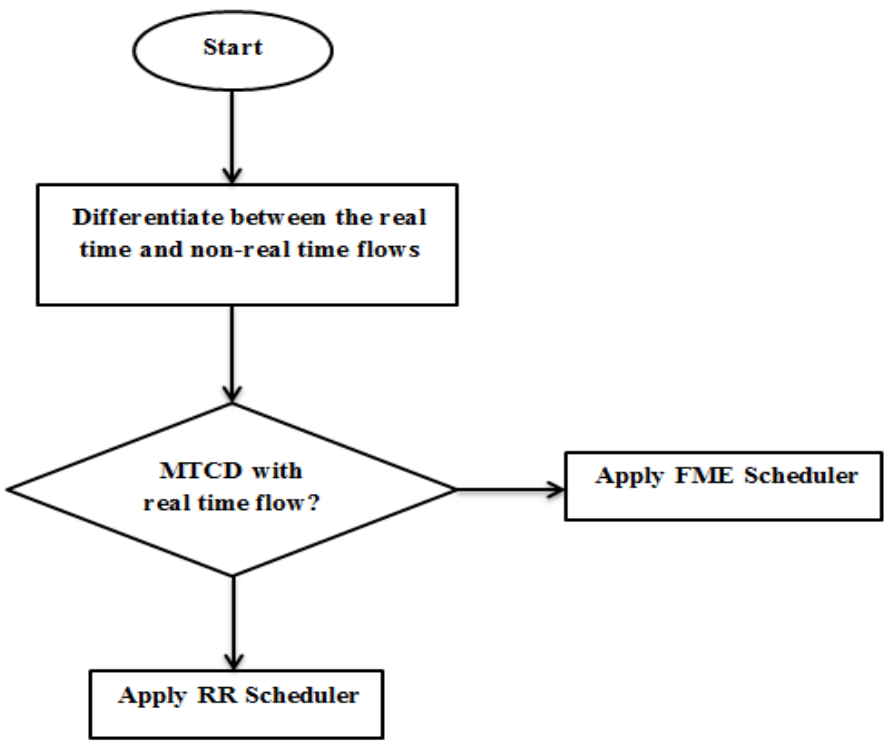

Figure 3. Flowchart of the proposed scheduler

\section{RESULTS AND ANALYSIS}

In this section, the uplink schedulers already mentioned and our proposal will be evaluated and compared using an open source simulator LTE-Sim [36]. To evaluate the QoS of a network, many parameters must be analyzed as throughput, goodput, fairness index, spectral efficiency and PLR [37]-[38].

\subsection{Simulation Environment}

The implementation of our proposed scheduler is made in a LTE uplink transmission for M2M communication developed with $\mathrm{C}++$ using the LTE-sim simulator; we evaluate the QoS support for uplink transmission based on priority and differentiation between heterogeneous traffic for exsiting uplink scheduling techniques and our proposed. 
In our simulation, we consider a single LTE cell, in which the users are uniformly distributed. In the centre of the cell, the eNodeB is positioned, whereas the users are modelled according to a random mobility model. In this paper, video and voip flows are used for real time services while infinite-buffer as known as Best effort (BE) flows represented the non-real time. The distribution of the users, in terms of traffic classes, is considered in our scenario as follows: $40 \%$ of users have video traffic flow, $40 \%$ users with voip traffic and $20 \%$ with best effort (infinite buffer). The purpose of this simulation is to evaluate the performance of the LTE network in high congestion, our evaluation is based on uplink schedulers implemented in the base stations of the LTE network which are RR, FME, MT and our proposed scheduler by the measurement of throughput, goodput, fairness, spectral efficiency and PLR, the simulation parameters are illustrated in the following Table 1 .

Table 1. Simulation Parameters

\begin{tabular}{cc}
\hline Simulation Parameters & Values \\
\hline System type & Single cell \\
Simulation duration & $120 \mathrm{~s}$ \\
Flow duration & $120 \mathrm{~s}$ \\
Frame structure & FDD \\
Mobility Model & Random direction \\
Cell radius & $1 \mathrm{Km}$ \\
System Bandwidth & $20 \mathrm{MHz}$ \\
RB Bandwidth & $180 \mathrm{kHz}$ \\
Time Slot & $0.5 \mathrm{~ms}$ \\
Scheduling Time (TTI) & $1 \mathrm{~ms}$ \\
Number of RBs & $100 \mathrm{RBs}$ \\
Maximum delay & $0.1 \mathrm{~s}$ \\
Video Bit Rates & $242 \mathrm{kbps}$ \\
VoIP Bit Rates & $8.4 \mathrm{kbps}$ \\
Number of MTCDs & 20 à 100 \\
\hline
\end{tabular}

\subsection{Evaluation Metrics}

\subsubsection{Throughput}

This is the total bit rate in reception; the measurement of this bit rate is one of the important operations which make it possible to identify the average success rate of transfer of the messages on a communication channel. Its value is calculated for a time interval, by dividing the total amount of information received during this interval, by the duration of the interval in question. The general formula for the calculation of the useful flow is:

$$
\text { Throughput }=\frac{\mathrm{Np} * \mathrm{Tp}}{\mathrm{T}}
$$

$\mathrm{Np}$ : is the number of packets received during the time interval

Tp: is the size of the package.

$\mathrm{T}$ : is the duration of the time interval.

\subsubsection{Goodput}

Goodput is the average rate of a successful transmission of data over a communication channel. In our simulation, this measure measures only the total data rate on the network, ignoring all other headers. A user's goodput is measured by first counting the total number of successfully received data packets and calculating the number of bits received, which is ultimately divided by the total execution time of the simulation. We measure application performance using goodput as follows:

$$
\text { Goodput }=\frac{\text { Original_Data }}{\text { Time }}
$$

Where original data is the number of bytes delivered at the receiver (that is, excluding retransmitted packets and headers), and Time is the amount of time required for the corresponding data delivery. We use average goodput to measure flow efficiency. The average goodput for $\mathrm{n}$ feed is defined as:

$$
\text { Average }_{\text {Goodput }}=\frac{\sum_{\mathrm{i}=1}^{\mathrm{n}}\left(\text { Goodput }_{\mathrm{i}}\right)}{\mathrm{n}}
$$


Where Goodputi is the goodput of the $\mathrm{i}$ flow and $\mathrm{n}$ the number of flows.

\subsubsection{Fairness Index}

Fairness Index is obtained by considering the rate achieved by each flow at the end of each simulation. Level of equity received by each flow according to Jain's Equity Index is given as follows:

$$
\text { Fairness }_{\text {index }}=\frac{\left(\sum \mathrm{x}_{\mathrm{i}}\right)^{2}}{\mathrm{n} \times \sum \mathrm{x}_{\mathrm{i}}^{2}}
$$

Where $x_{i}$ is the user's rate $i$ and $n$ is the number of enabled flows

\subsubsection{Spectral Efficiency}

Spectral efficiency is defined as the maximum user rate divided by the bandwidth of the channel. This is the number of received bits correctly normalized by the resource consumed in time and bandwidth. Thus, spectral efficiency is strongly related to resource consumption and packet error rate.

$$
\text { Spectral Efficiency }=\frac{\mathrm{D}_{\max }}{\mathrm{w}_{\mathrm{c}}}
$$

With $\mathrm{D}_{\max }$ is the maximum rate of the user and $\mathrm{W}_{\mathrm{c}}$ the bandwidth of the channel.

\subsubsection{Packet Lost Rate}

Improvement in Packet Loss Rate (PLR) estimation is a crucial issue, because its value has a big effect on network performance, especially when it comes to real-time traffic like voip and video streams. Packet loss is the lost bytes when transmitting packets. It expresses itself in rate of loss. This can be caused by many factors, mostly due to network congestion, as it can be caused by packet latency. The rate of lost packets is calculated as follows:

$$
\mathrm{PLR}=\frac{\mathrm{N}_{\mathrm{PL}}}{\mathrm{N}_{\mathrm{PS}}} * 100
$$

NPL: is the number of packets lost.

NPS: is the number of packets sent.

\subsection{Performance Evaluation}

The average throughput of RR, FME, MT and proposed scheduler versus a different number of MTCDs is shown in Figure 4. This figure shows that the throughput achieved by the proposed method is better than other schemes. In fact, the proposed scheme tries to maximize total throughput. So system throughput of the proposed increases when the number of MTC devices rises.

The results about average goodput indicate that the proposed scheme followed by FME are the best compared to RR and MT for mixed traffic in Figure 5.



Figure 4. Average Throughput

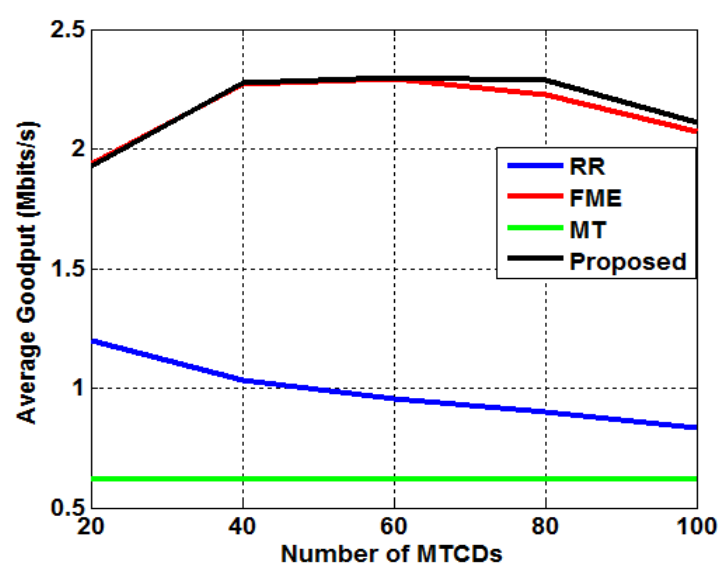

Figure 5. Average Goodput 
Figure 6 shows the results in terms of fairness for the RR, FME, MT and our proposed scheduler. The results about Fairness Index indicate that RR is the best scheduler among them because RR does not depend on any channel feedback to assign RBs to MTCDs as others require. RR should have an ideal value of one, since it is fairly serving all MTCDs. The RR scheduler assigns PRBs cyclically so that the same throughput is guaranteed for all devices, that way Jain's fairness index is maximize. Herein we use this index as the measurement criterion for data-rate fairness. The simulation shows that the proposed scheme achieved more interesting results than other schedulers compared to RR scheme.

As shown in Figure 7, the MT scheduler has a better spectral efficiency compared to that measured for the other scheduler because among the strong points of MT it allows to improve spectral efficiency, despite this it starts decreasing when we reach a large number of devices in our simulation it's 80 MTCDs. For our proposed scheduler we can see that have a high efficiency and the value of spectral efficiency increases as the number of devices increases.

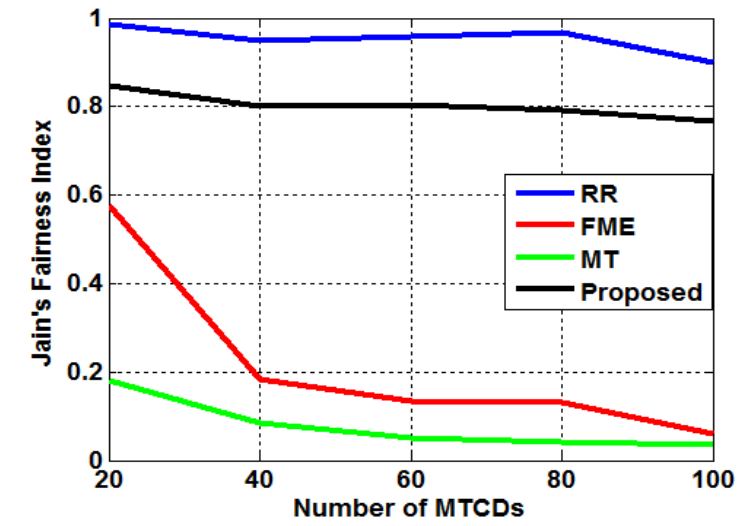

Figure 6. Fairness index

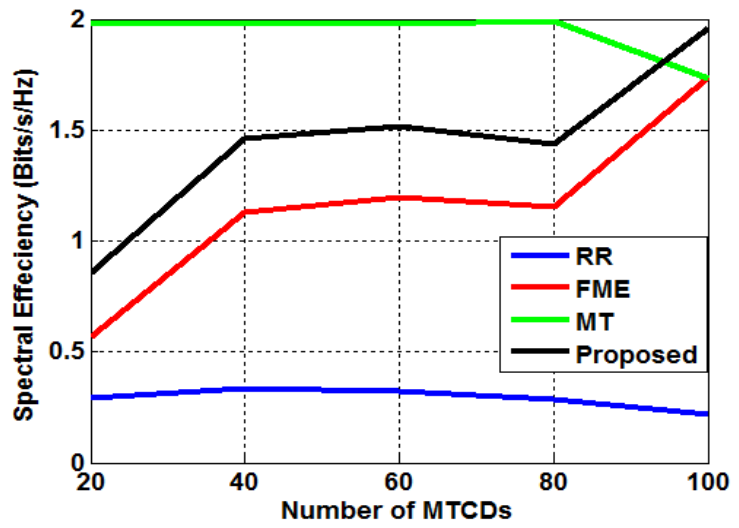

Figure 7. Spectral efficiency

Figure 8 represents the evolution of the packet lost rate as a function of the number of MTCDs in the coverage area of the cell, when the number of active devices increases in the cell, the loss rate increases, in particular for the FME algorithm which reach a maximum rate. As shown in the figure, it can be seen that our proposed scheduler has the lowest PLR this is due to the combination between two different schedulers that takes advantage of their benefits in order to achieve maximum throughput and less packet lost rate.



Figure 8. Packet Lost Rate (PLR)

\section{CONCLUSION}

Packet scheduling and distributing resources among users is still the main challenge due to unfairness and low performance which occur when allocating resources to users. In this paper, the above mentioned challenges are studied and analyzed, focusing on three schedulers; they are RR, FME and MT. 
These methods do not provide QoS to users that use different types of traffic flows. The proposed algorithm in this paper is to combine the RR scheduler and the FME scheduler in order to fulfil the QoS criteria maximizing throughput and minimizing the packet loss rate for real time service. Voip and video have been selected as real time traffic and best effort as non-real time. LTE-Sim simulator is used to compare between the mentioned schedulers and our propoed in terms of throughput, goodput, fairness index, packet loss ratio and spectrum efficiency. This new scheduler is considered as QoS scheduler that supports real and non-real time service in LTE Network. The main key for this scheduler is to maximize the throughput and to ensure the fairness among users with higher priority for real time. The newly proposed scheduler shows the best result among others where higher priority is given to the real time traffic flows (voip, video).

\section{REFERENCES}

[1] L. Atzori, et al., "The Internet of Things: A survey, " Computer Networks, pp.2787-2805, 2010.

[2] A. Rghioui and A. Oumnad, "Internet of Things: Surveys for Measuring Human Activities from Everywhere," International Journal of Electrical and Computer Engineering (IJECE), vol/issue 7(5), pp. 2474-2482, 2017.

[3] J. Kim, et al., "M2M service platforms: Survey, issues, and enabling technologies," IEEE Communications Surveys Tutorials, vol.16, no.1, pp.61-76, 2014.

[4] T. Taleb and A. Kunz, "Machine type communications in 3GPP networks: Potential, challenges and solutions," Communications Magazine, vol.50, no.3, pp.178-184, 2012.

[5] A. Biral, et al., "The challenges of M2M massive access in wireless cellular networks," Digital Communications and Networks, 2015.

[6] A. Aijaz, et al., "On Radio Resource Allocation in LTE Networks with Machine-to-Machine Communications," in 2013 IEEE 77th Vehicular Technology Conference (VTC Spring), 2014.

[7] M. Ouaissa, et al., "Analysis of Authentication and Key Agreement (AKA) Protocols in Long Term Evolution (LTE) Access Network, " in 1st Springer International Conference on Emerging Trends and Advances in Electrical Engineering and Renewable Energy, 2016.

[8] M. Ouaissa, et al., "Impact of M2M Traffic in Random Access Channel over LTE Networks," in 1st Springer International Conference on Emerging Trends and Advances in Electrical Engineering and Renewable Energy, 2016.

[9] M. Ouaissa, et al., "Performance Analysis of Random Access Mechanisms for Machine Type Communications in LTE Networks," in Int. Conf. on Advanced Communication Systems and Information Security 2016 (ACOSIS'16), 2016.

[10] A. E. Mostafa and Y. Gadallah, "A Statistical Priority-Based Scheduling Metric for M2M Communications in LTE Networks," IEEE ACCESS, vol. 5, 2017.

[11] J. Ding and N. Saxena, "SMART M2M UPLINK SCHEDULING ALGORITHM OVER LTE," International Journal of Advanced Computational Engineering and Networking, vol.2, no.5, 2014.

[12] S. Zhenqi, et al., "Research On Uplink Scheduling Algorithm Of Massive M2M And H2H Services In LTE," in Proc. of IET International Conference on Information and Communications Technologies (IETICT 2013), 2013.

[13] J. Brown and Jamil Y Khan, "Predictive Resource Allocation in the LTE Uplink for Event Based M2M Applications," in Proc. of IEEE International Conference on Communications Workshops (ICC), 2013.

[14] Y. B. Chen, et al., "An Energy-Efficient Scheduling Algorithm for Real-Time Machine-to-Machine (M2M) Data Reporting," in Proc. of IEEE Global Communications Conference, 2014.

[15] A. Elhamy and Y. Gadallah, "BAT: A Balanced Alternating Technique for M2M Uplink Scheduling over LTE," in Proc. of IEEE 81st Vehicular Technology Conference (VTC Spring), 2015.

[16] M. K. Giluk, et al., "Class Based Dynamic Priority Scheduling for Uplink to Support M2M Communications in LTE," in Proc. of IEEE World Forum on Internet of Things (WF-IoT), 2014.

[17] I. Abdalla and S. Venkatesan, "A QoE preserving M2M-aware hybrid scheduler for LTE uplink," in Proc. of International Conference on Selected Topics in Mobile and Wireless Networking (MoWNeT), 2013.

[18] A.S. Lioumpas and A. Alexiou, "Uplink scheduling for Machine-to-Machine communications in LTE-based cellular systems," in Proc. of IEEE GLOBECOM Workshops (GC Wkshps),2011.

[19] F. Ghavimi and H. H. Chen, "M2M Communications in 3GPP LTE/LTE-A Networks: Architectures, Service Requirements, Challenges, and Applications," IEEE Communications Surveys Tutorials, 2014.

[20] M. Ouaissa, et al., "Group Access Authentication of Machine to Machine Communications in LTE Networks," in The second International Conference on Internet of Things, Data and Cloud Computing (ICC 2017), 2017.

[21] M. Ouaissa, et al., "New Method to Control Congestion for Machine to Machine Applications in Long Term Evolution System," International Journal on Communications Antenna and Propagation (I.Re.C.A.P.), vol. 8, no. 4, 2018.

[22] M. Ouaissa and A. Rhattoy, "A New Scheme of Group-based AKA for Machine Type Communication over LTE Networks," International Journal of Electrical and Computer Engineering (IJECE), vol. 8, no. 2, pp. 1169-1181, 2018.

[23] G. Fritze, "SAE: The Core Network for LTE," Ericsson, Technical white paper, 2012.

[24] S. Sesia, et al., "LTE-The UMTS Long Term Evolution: From Theory to Practice," 2nd edition, 2011.

[25] M. Coupechoux, and P. Martins, "Vers les systèmes radio mobiles de 4e génération - de l'UMTS au LTE," 2013.

[26] 3GPP TS 22.368. V14.0.1, "Service requirements for Machine-Type Communications (MTC); Stage 1 (Release 14)," 2017.

QoS hybrid uplink scheduler based on service type for M2M communications in LTE ... (Mariyam Ouaissa) 
[27] M. Ouaissa and A. Rhattoy, "New Method Based on Priority of Heterogeneous Traffic for Scheduling Techniques in M2M Communications over LTE Networks," International Journal of Intelligent Engineering and Systems, vol.11, no.6, 2018.

[28] M. Ouaissa, et al., "Comparative Performance Study of QoS Downlink Scheduling Algorithms in LTE System for M2M Communications," in 2nd International Conference Europe Middle East \& North Africa Information Systems and Technologies to support Learning (EMENA-ISTL 2018), 2018.

[29] Tiago P. C. de Andrade Tiago, et al., "Allocation of Control Resources for Machine-to-Machine and Human-toHuman Communications Over LTE/LTE-A Networks," IEEE INTERNET OF THINGS JOURNAL, vol. 3, no. 3, pp. 366-377, 2016.

[30] K. Zheng, et al., "Radio Resource Allocation in LTE-A Cellular Networks with M2M Communications," IEEE Commun. Mag.,2012.

[31] 3GPP TS 36.300. V15.2.0, "Evolved Universal Terrestrial Radio Access (E-UTRA) and Evolved Universal Terrestrial Radio Access Network (E-UTRAN); Overall description; Stage 2 (Release 15)," 2018.

[32] 3GPP TS 36.211. V8.0.0, "Evolved Universal Terrestrial Radio Access (E-UTRA); Physical Channels and Modulation," 2018.

[33] 3GPP TS 36.321. V15.2.0, "Evolved Universal Terrestrial Radio Access (E-UTRA); Medium Access Control (MAC) protocol specification Release 15)," 2018.

[34] Y. Bouguen, E. Hardouin, and F.X. Wolff, LTE et les réseaux 4G, 2012.

[35] S. B. Ismail, et al., "Performance Analysis of Uplink Scheduling Algorithms in LTE Networks, " Indonesian Journal of Electrical Engineering and Computer Science, vol. 9, no. 2, pp. 373-379, 2018.

[36] G. Piro, et al., "Simulating LTE Cellular Systems: an Open Source Framework," IEEE Transactions on Vehicular Technology, vol.60, no.2, pp.498-513, 2010.

[37] S. A. AlQahtani and M. Alhassany, "Performance Modeling and Evaluation of Novel Scheduling Algorithm for LTE Networks," Cyber Journals: Multidisciplinary Journals in Science and Technology, Journal of Selected Areas in Telecommunications (JSAT), vol.4, no.1, 2014.

[38] A.M. Sahibzada, et al., "Fairness Evaluation of Scheduling Algorithms for dense M2M Implementations," in Proc. of IEEE WCNC 2014 - Workshop on IoT Communications and Technologies, 2014. 